Gesnerus 57 (2000) 182-205

\title{
Hilberts Ruf nach Bern
}

Tilman Sauer*

\section{Summary}

Attempts to find a successor for the late Berne professor of mathematics Johann Heinrich Graf in winter and spring 1919 proved fruitless when, unexpectedly, the renowned Göttingen mathematician David Hilbert intimated that he himself would, under certain conditions, accept a call to Berne. Subsequent negotiations between Hilbert and the Bernese and Prussian authorities mainly concerned the question of salary, with offers of unusually high compensation both in Berne and in Göttingen. Although Hilbert eventually declined for financial reasons, it is argued that his considerations may well have been rather complex and reflect both his own situation in Göttingen and the general state of affairs of university mathematics in Germany and Switzerland after the First World War.

\section{Zusammenfassung}

Nach erfolglosen Versuchen im Winter und Frühjahr 1919, einen Nachfolger für den verstorbenen Berner Ordinarius für Mathematik Johann Heinrich Graf zu finden, liess unerwarteterweise der bekannte Göttinger Mathematiker David Hilbert durchblicken, dass er selbst unter bestimmten Bedingungen bereit wäre, einen Ruf nach Bern anzunehmen. Obwohl es in den nachfolgenden Verhandlungen Hilberts mit den Berner und Preussischen Unterrichtsbehörden vornehmlich um Gehaltsfragen ging und Hilbert nach ungewöhnlich grosszügigen Angeboten aus Bern und Berlin den Berner Ruf schliesslich ablehnte, mögen Hilberts Überlegungen dennoch komplex

* Ich danke Robert Casties, Hans-Joachim Dahms, Gerd Grasshoff, Andrea Loettgers, Kärin Nickelsen, Franziska Rogger und Klaus Sommer für Anregungen, Hinweise und Kommentare.

Tilman Sauer, Universität Bern, Institut für Exakte Wissenschaften, Sidlerstr. 5, CH-3012 Bern (tilman.sauer@philo.unibe.ch). 
gewesen sein. Sie spiegeln sowohl seine eigene damalige Situation in Göttingen wider als auch die allgemeine Situation der universitären Mathematik in Deutschland und in der Schweiz nach dem Ersten Weltkrieg.

\section{Die Vakanz}

Am 17. Juni 1918 starb, 65jährig, der Berner Ordinarius für Mathematik Johann Heinrich Graf an einem Schlaganfall. Mit seinem Tod wurde eine der beiden mathematischen Professuren der Berner Hochschule vakant, die Graf 26 Jahre lang bekleidet hatte, nachdem er 1892 zum Nachfolger Ludwig Schläflis berufen worden war. Grafs unerwarteter Tod riss eine spürbare Lücke in den mathematischen Lehrbetrieb der Universität, die möglichst umgehend wieder geschlossen werden musste.

Bis zu seinem Ableben hatte sich Graf die Last der mathematischen Ausbildung ${ }^{1}$ vor allem mit seinen beiden Kollegen, dem Ordinarius für Mathematik und höhere Geometrie Jaques Louis Crelier (1873-1935) und dem Ordinarius für Mathematik und theoretische Astronomie Gottlieb Huber (1857-1923), geteilt. Über mathematische oder verwandte Gebiete lasen darüber hinaus zwei Privatdozenten, dann der Versicherungsmathematiker Christian Moser (1861-1935) und schliesslich Paul Gruner (1869-1957), ordentlicher Professor für theoretische Physik. Für das laufende Semester hatte Graf insgesamt 22 Wochenstunden Vorlesungen angekündigt ${ }^{2}$. Er las über Differential- und Integralrechnung, Kugelfunktionen, Bessel'sche Funktionen, Funktionentheorie, Differentialgleichungen und über Rentenund Versicherungsrechnung. Zusätzlich leiteten Graf und Huber das mathematische Seminar ${ }^{3}$.

Mit dem Ausfall eines Grossteils des mathematischen Lehrangebots unerwartet konfrontiert, beschloss die II. Abteilung der Philosophischen Fakultät jedoch, mit der Neubesetzung auf jeden Fall noch bis zum Sommer 1919 zu warten ${ }^{4}$. Die wichtigsten ausgefallenen Vorlesungen sollten vertretungsweise Huber, Moser und einer der Privatdozenten übernehmen, während eine vom Dekan geführte Kommission mit den Herren Huber, Moser,

1 Vgl. zum folgenden Vorlesungen Bern (1918), Verzeichnis Bern (1918), sowie Feller (1935) 546-548, Gorgé (1984) und Kommission für bernische Hochschulgeschichte (1984a).

2 Vgl. Vorlesungen Bern (1918) 17.

3 Grafs Vorlesungen waren von durchschnittlich acht bis neun Studenten besucht worden, nur für die Bessel'schen Funktionen hatten sich lediglich vier Zuhörer und für das mathematische Seminar 17 Teilnehmer interessiert. Vgl. seine Mitteilung über die abgehaltenen Vorlesungen in SAB BBIIIb 1099, B1. 113, und die Kolleggeldnachweise im Hauptbuch des Quästors in SAB BBIIIb 873, Bl.349-356.

4 PFB Protokollbuch, Bd. XI, pp. 226f., Sitzung vom 8. Juli 1918. 
Crelier und Gruner die Frage der Neubesetzung vorbereitete. Nach den Semesterferien berichtete Professor Huber der Abteilung über die bisherigen Beratungen der Kommission und beantragte eine «ordnungsgemässe» Ausschreibung der Professur zur Neubesetzung auf den 1. April 1919. Dem Antrag der Kommission folgend beschloss die Fakultät, die Ausschreibung solle für «Mathematik, und zwar Analysis, besonders Funktionentheorie» erfolgen, man wolle die Entscheidung, ob eine ordentliche oder eine ausserordentliche Professur zu besetzen sei, erst nach Massgabe der eingegangenen Bewerbungen fällen, und es sollten womöglich Schweizer Bewerber bevorzugt werden 5 . Der gewünschte Schwerpunkt der Ausschreibung, so berichtete der Dekan dem Direktor des Unterrichtswesens Leo Merz ${ }^{6}$, ergebe sich aus Grafs früherem Lehrauftrag, denn da die Mathematik «in zwei Hauptteile, nämlich Analysis und Geometrie» zerfiele und die Vertretung der Analysis nunmehr «erloschen» sei, sei nun die Stelle «mit einem wirklich hervorragenden, mit der modernen Mathematik völlig vertrauten Fachmanne» neu zu besetzen. Es käme jetzt alles darauf an, «die Gelegenheit, der bernischen Mathematik neues Blut zuzuführen, nicht zu versäumen».

Wie von der Fakultät beantragt, wurde die vakante Professur wenige Tage später ausgeschrieben, und zwar in knapp einem Dutzend sowohl schweizerischen, deutschen als auch französischen Zeitungen ${ }^{7}$. Aber obwohl die Vakanz damit einem breiten Publikum bekannt gemacht worden war und bis zum Ende der Ausschreibungsfrist am 15. Januar insgesamt 14 Bewerbungen eingegangen waren ${ }^{8}$, war das Ergebnis der Ausschreibung für die Kommission unbefriedigend.

Unter den eingegangenen Bewerbern, so führt ein Bericht der Kommission vom 24. Februar9 aus, seien lediglich drei Schweizer gewesen, die aber allesamt nicht in Frage kämen. Einer von diesen war Ferdinand Gonseth (1890-1975), Privatdozent für angewandte Mathematik an der Universität Zürich, der von seiner fachlichen Ausrichtung her nicht in Betracht kam, da er Geometer war und man für die Professur einen Analytiker suchte. Über die eingegangenen Bewerbungen hinaus hatte die Kommission sich noch

5 PFB Protokollbuch, Bd. XI, p. 240.

6 Volkmar Kohlschütter an Leo Merz, 20. November 1918, in SAB BBIIIb 615, Nr. 11.

7 Die Anzeige erschien in folgenden Zeitungen: Amtliches Schulblatt, Amtsblatt, Der Bund, Neue Zürcher Zeitung, Münchner Hochschulnachrichten, Frankfurter Zeitung, Journal de Genève, Enseignement mathematique, Le Democrate, Le Pays; vgl. die Liste in SAB BBIIIb 615, Nr. 11.

8 Die Bewerbungsunterlagen sind in der Akte SAB BBIIIb 615, Nr. 11 erhalten.

9 Die II. Abteilung der Fakultät bestätigte diesen Bericht und leitete ihn zwei Tage später an die Direktion des Unterrichtswesens weiter. PFB Protokollbuch, Bd. XI, pp. 258f.; SAB BBIIIb 615, Nr. 11. 
selbst an den schweizerischen Hochschulen nach einem geeigneten Kandidaten umgesehen, aber zwei in Betracht gezogene Mathematiker hatten aus Rücksicht auf die Hochschulen, denen sie damals angehörten, einen Ruf nicht in Erwägung ziehen wollen.

Unter den ausländischen Bewerbern nennt der Bericht zunächst einen der Berner Privatdozenten, der aber ebenfalls nicht empfohlen werden konnte, da er Geometer war. Von den verbleibenden 10 Kandidaten kamen für die Kommission von ihren wissenschaftlichen Leistungen nur drei in Betracht ${ }^{10}$. Zunächst Karl Boehm (1873-1958), ordentlicher Professor an der technischen Hochschule in Karlsruhe, dann Alfred Löwy (1873-1935), ordentlicher Honorarprofessor in Freiburg, und schliesslich Georg Pólya (1887-1985), Privatdozent für Mathematik an der ETH Zürich. Von diesen könne Löwy nicht empfohlen werden, da er nach den Erkundungen der Kommission «als Hochschullehrer ganz unglücklich, ohne Erfolg und Anregung bei den Studenten» sei. Über Boehm teilt der Bericht viel Positives mit, allerdings seien seine wissenschaftlichen Arbeiten «sehr verschieden beurteilt». Wissenschaftlich «der bedeutendste aller Bewerber» schliesslich sei Georg Pólya. Er habe «über 50 mathemat. Arbeiten in deutschen, französischen, ungarischen und italienischen mathemat. Zeitschriften publiziert, die von Wichtigkeit für die Analysis sind».

Interessanterweise findet sich in der Bewerbungsakte von Pólya, der nach seiner Promotion von Herbst 1912 bis Frühjahr 1914 für drei Semester in Göttingen gewesen war, ein Empfehlungsschreiben aus Göttingen mit Datum vom 7. Dezember 1918 und unterschrieben von «Professor D. Hilbert, Professor C. Runge, Professor E. Landau, Professor Hecke». In diesem Gutachten, um das Pólya «einen der Unterzeichneten» gebeten hatte, wird das uneingeschränkte Lob der wissenschaftlichen und akademischen Leistungen Pólyas schliesslich so zusammengefasst:

Natürlich wissen wir nicht, wer sich etwa noch bewirbt; unser Gutachten soll sich daher gegen keinen bestimmten anderen richten. Unter den jüngeren Mathematikern in der Schweiz wüssten wir allerdings keinen, der ihm überlegen oder auch nur annähernd gleichwertig wäre.

Trotz diesem gewichtigen, wenn auch recht selbstbewussten Urteil hatte die Kommission allerdings offensichtlich weitere, unabhängige Erkundungen über Pólya eingeholt:

Die Urteile über seine persönlichen Eigenschaften lauten [...] ziemlich übereinstimmend, aber nicht gerade günstig, so dass wir bezweifeln müssen, einen angenehmen Kollegen in ihm zu erhalten. Die Ansichten über seine Lehrbefähigung sind geteilt. Herr Polya ist semitischer Abstammung, ungarischer Refraktär [i.e. Kriegsdienstverweigerer, T.S.] und seit Frühjahr 1918 Schweizerbürger.

10 Die nicht berücksichtigten Bewerber waren zum überwiegenden Teil nicht habilitierte Gymnasiallehrer. 
Mit diesen Beurteilungen kommt der Bericht schliesslich zu dem zusammenfassenden Ergebnis,

dass die Qualifikation der angemeldeten Bewerber für die zu besetzende Math.professur die Fakultät entweder in wissenschaftlicher Hinsicht oder in Bezug auf Lehrbefähigung und persönliche Eigenschaften, nicht voll befriedigt hat.

In Betracht kämen für die Stelle daher nur, an erster Stelle, Boehm für ein Ordinariat, und, an zweiter Stelle, Pólya für ein Extraordinariat. Als erwägenswerte alternative Möglichkeit, die Frage der Nachfolge Graf zu regeln, wurde aber auch zu bedenken gegeben, Crelier, der sich bereits früher «vorwiegend mit Analysis beschäftigt» habe, den Lehrauftrag Grafs zu übertragen, in welchem Falle dann dessen Lehrauftrag für Geometrie neu besetzt werden müsste.

In dem Begleitschreiben dieses Berichts an die Unterrichtsdirektion wurde zur näheren Erörterung um eine mündliche Unterredung ersucht. Was immer deren Inhalt gewesen sein mag, weder erfolgte ein Ruf an Boehm oder Pólya noch wurde der Lehrauftrag Crelier übertragen und ein weiterer Geometer gesucht. Statt dessen sah sich die Kommission informell und zunächst weiterhin vergeblich nach weiteren Analytikern um.

Am 28. Juni 1919, dem Tag der Unterzeichnung des Versailler Friedensvertrages, schliesslich schrieb Huber an den Direktor des Unterrichtswesens Merz einen Brief, in dem er die bisherigen Bemühungen zusammenfasste, einen Nachfolger für die Professur Graf zu finden ${ }^{11}$. Da für die Stelle ein geeigneter Schweizer Kandidat nicht zu finden war, sei man seit letztem Winter unablässig bemüht gewesen, einen passenden deutschen oder österreichischen Kandidaten ausfindig zu machen. Diese Suche sei erschwert durch die derzeitigen «schwierigen Verkehrsverhältnisse», die zur Folge hätten, dass Korrespondenz regelmässig erst nach zwei bis vier Wochen einträfe. Was ausländische deutsche Kandidaten angehe, habe ihm sein «alter Lehrer Pringsheim» aus München geschrieben,

dass fast alle einigermassen anerkannten deutschen Mathematiker gegenwärtig auf guten, vielfach auf ausgezeichneten Ordinariaten sitzen $u$. für uns kaum in Betracht kommen dürften, zumal bei den jetzigen ungeheuren Schwierigkeiten des Auswanderns aus Deutschland (Steuerflucht).

\section{Der Kandidat}

Unter den 14 Bewerbungen für die im November 1918 ausgeschriebene Berner Professur für Mathematik war ein Gutachten David Hilberts für den

11 Huber an Merz, 28. Juni 1919, in SAB BBIIIb 616, Nr. 16 (Akte Hilbert). 
Zürcher Bewerber Georg Pólya, nicht aber eine eigene Bewerbung des Göttinger Mathematikers eingegangen.

Hilbert, 1862 in Königsberg geboren, war zum Zeitpunkt der Ausschreibung 56 Jahre alt ${ }^{12}$. Nachdem er Kindheit, Schule, Studium und - bis auf eine halbjährige Auslandsreise - auch seine frühe akademische Karriere mit Promotion, Habilitation, Berufung zum Extraordinarius und zum Ordinarius in Königsberg verbracht hatte, war er 1895 auf Betreiben Felix Kleins (1849-1925) auf die zweite ordentliche Professur für Mathematik in Göttingen berufen worden. Diese Berufung erfolgte in dem Bemühen Kleins und Friedrich Althoffs (1839-1908), Göttingen zu einem Zentrum der Mathematik in Preussen auszubauen ${ }^{13}$. Tatsächlich war Hilberts Berufung nach Göttingen in dieser Hinsicht ein durchschlagender Erfolg. Schon seine frühen Arbeiten über Invariantentheorie ${ }^{14}$ hatten Aufsehen erregt, in Göttingen folgte dann sein monumentaler, im Auftrag der Deutschen Mathematiker-Vereinigung verfasste Bericht über die «Theorie der algebraischen Zahlkörper» ${ }^{15}$. Im Jahre 1900 war Hilbert Vorsitzender der Deutschen Mathematikervereinigung und hielt als solcher auf dem zweiten internationalen Mathematikerkongress in Paris einen programmatischen Vortrag über «Mathematische Probleme», in dem er seine berühmt gewordenen 23 Probleme der mathematischen Forschung des 20. Jahrhunderts formulierte ${ }^{16}$. Sein epochemachendes Werk über die «Grundlagen der Geometrie» von $1899^{17}$ begründet das moderne Verständnis mathematischer Axiomatik, geprägt durch die Forderung nach Unabhängigkeit, Konsistenz und Vollständigkeit von Axiomensystemen, durch den Begriff der impliziten Definition sowie den Gebrauch von Modellen in der logischen Analyse von Axiomensystemen. Hinzu kommen Arbeiten zur Variationsrechnung und zum Dirichletschen Prinzip sowie die Begründung einer umfassenden Theorie linearer Integralgleichungen ${ }^{18}$. 1909 löste Hilbert das bereits 1782 formulierte Waringsche Problem. Für seine mathematischen Arbeiten wurde

12 Zur Biographie Hilberts vgl. Blumenthal (1935), Reid (1970).

13 Vgl. hierzu Brocke (1980) und Tobies (1991), eine allgemeine Charakterisierung der Mathematik in Göttingen zur Zeit Hilberts und Kleins gibt Rowe (1989).

14 Die meisten von Hilberts publizierten Arbeiten sind in einer noch zu seinen Lebzeiten veröffentlichten, dreibändigen Ausgabe seiner Gesammelte(n) Abhandlungen zugänglich (Hilbert [1932-1935]), eine mehrbändige Edition ausgewählter, unpublizierter Schriften zu den Grundlagen der Mathematik und der Naturwissenschaften wird derzeit in Göttingen vorbereitet, vgl. http://www.gwdg.de/ uhwg/Hilbert-Edition.html.

15 Hilbert (1932-1935) Bd. 1,63-363, von dieser Arbeit ist kürzlich eine englische Übersetzung erschienen: Hilbert (1998).

16 Hilbert (1932-1935) Bd.3,290-329, über die spätere Bedeutung der Hilbertschen Probleme vgl. Bieberbach (1930), Wussing (1976).

17 Hilbert (1999).

18 Hilbert (1912). 
Hilbert 1910 der Bolyai-Preis verliehen ${ }^{19}$. Seit 1911 hatte Hilbert sich verstärkt mit mathematischer Physik beschäftigt und auch auf diesem Gebiet publiziert, so 1915 eine Arbeit über die «Grundlagen der Physik», in welcher er zeitgleich mit Einstein die korrekten Gravitationsgleichungen der allgemeinen Relativitätstheorie publizierte, indem er diese mittels der Variation der Riemannschen Krümmung einführte ${ }^{20}$.

Der Erfolg und die Vielseitigkeit der Hilbertschen Arbeiten schlugen sich auch in zahlreichen Anerkennungen und Auszeichnungen nieder ${ }^{21}$. Er war auswärtiges oder korrespondierendes Mitglied der wissenschaftlichen Akademien in Uppsala (1903), Turin (1903), Rom (1903), Bologna (1904) München (1905), Kopenhagen (1906), Oslo (1906), Venedig (1910), Stockholm (1912), Brüssel (1912), Berlin (1913), Helsinfors (1914) sowie auswärtiges Mitglied der National Academy of Sciences of the United States (1907). Hinzu kamen eine Ehrendoktorwürde in Oslo 1902 sowie die Ernennung zum Geheimen Regierungsrat durch eine kunstvoll von Kaiser Wilhelm persönlich unterzeichnete Urkunde vom November 190422 .

Der stetig wachsende Ruhm Hilberts trug zum stetig wachsenden Ruhm Göttingens als eines Zentrums mathematischer Forschung bei. Die Verbindung Hilberts mit Göttingen war dabei bereits mehrfach durch Rufe an Hilbert auf auswärtige Professuren auf die Probe gestellt worden. Alle diese Rufe hatte Hilbert abgelehnt und sich in den Bleibeverhandlungen ein in jeder Hinsicht vorteilhaftes, um nicht zu sagen «ausgezeichnetes» deutsches Ordinariat ausgehandelt.

Ein 1898 ergangener Ruf an die Universität Leipzig als Nachfolger von Sophus Lie brachte ihm eine deutliche Gehaltserhöhung seines Jahresgehalts von 4000 M um 2000 M jährlich, zuzüglich einer «Aktivitätszulage», d.h. einer «bei seiner eventuellen Emeritierung in Wegfall kommende Zulage» von $1000 \mathrm{M}^{23}$.

19 Reid (1970) 106, 125, für eine Gesamtwürdigung des mathematischen Werks Hilberts vgl. Weyl (1944).

20 Hilbert (1915). Über die historische Bewertung dieser Arbeit hat es in letzter Zeit eine ausführliche Debatte gegeben, siehe Corry (1999), Renn/Stachel (1999), Sauer (1999) sowie weitere in diesen Arbeiten zitierte Literatur.

21 Vgl. die entsprechenden Urkunden in SUB Cod. Ms. Hilbert 742 und 743.

22 Wie zusätzlich aus der Personalakte ersichtlich ist, hatte der Kaiser Hilbert bereits 1901 den «Rothen Adlerorden vierter Klasse» verliehen. Ausserdem erhielt Hilbert 1907 den Königlichen Kronenorden III. Klasse, und 1908 wurde ihm durch den Prinz-Regenten von Bayern der Maximilians-Orden für Wissenschaft und Kunst verliehen, vgl. UAG PA Hilbert, Bl. 16, 25-30.

23 UAG PA Hilbert, Bl. 14f.; über Hilberts Ruf nach Leipzig vgl. ferner (u.a.) den Berufungsvorschlag im Universitätsarchiv Leipzig PA583, B1. 13-16. Über Hilberts Einkünfte und Vermögensverhältnisse überhaupt vgl. SUB Cod. Ms. Hilbert 748. Über die Besoldung der preussischen Professoren allgemein vgl. auch Brocke (1980) 64. 
Einen 1902 erhaltenen Ruf nach Berlin als Nachfolger von Lazarus Fuchs (1833-1902) nutzte Hilbert, um in den Bleibeverhandlungen durchzusetzen, dass in Göttingen eine dritte ordentliche Professur für Mathematik eingerichtet und mit seinem langjährigen Freund Hermann Minkowski (18641909) besetzt wurde ${ }^{24}$. Diese Entscheidung des preussischen Kultusministeriums war ein weiterer Schritt im Ausbau Göttingens zu einem mathematischen Zentrum. Drei mathematische Ordinarien an einer Fakultät waren an preussischen Universitäten und an deutschsprachigen Universitäten überhaupt sehr selten ${ }^{25} .1904$ kam dann sogar noch ein viertes Ordinariat hinzu, das erste für angewandte Mathematik in Deutschland überhaupt, das mit Carl Runge (1856-1927) ausgezeichnet besetzt wurde.

Die Verstärkung des Göttinger mathematischen Lehrkörpers durch Runge und die ebenfalls 1904 erfolgte Berufung Ludwig Prandtls (18751953) zum Extraordinarius für technische Mechanik waren dann auch der Grund für Hilbert, einen von Leo Königsberger (1837-1921) im Jahre 1904 erwirkten Ruf nach Heidelberg, ohne zu zögern, abzulehnen ${ }^{26}$. Statt dessen erreichte Hilbert in diesbezüglicher Korrespondenz mit Althoff eine Umwandlung seiner seit dem Berliner Ruf als ausserordentliche Remuneration gewährten Gehaltserhöhung in ein festes Gehalt sowie eine Erhöhung seiner «Aktivitätszulage» auf $2900 \mathrm{M}^{27}$.

24 Über den Berliner Ruf von 1902 vgl. u.a. die Unterlagen im Archiv der Humboldtuniversität Berlin (Phil. Fak. 1463, Bl. 330-343), den Nachruf von Carathéodory (Sommerfeld/Carathéodory (1943), Biermann (1988), Reid (1970) 89 sowie Hilberts eigene Darstellung in seinem Nachruf auf Minkowski (Hilbert [1932-1935] Bd. 3, 355).

25 Vgl. hierzu Scharlau (1989).

26 Vgl. den Briefentwurf Hilberts an Königsberger vom 30. September 1899 sowie die Notiz über einen früheren Brief in SUB Cod. Ms. Hilbert 741/6: «Unter den gegenwärtigen Umständen, wo wir eben Runge u. Prandtl bekommen haben (zu Minkowski hinzu) war es für mich gar nicht anders möglich, als sofort abzulehnen»; sowie den Briefentwurf Hilberts an Althoff vom 14. August 1904 in SUB Cod. Ms. Hilbert 4/12: «... beeile ich mich, Ihnen mitzuteilen, dass ich den Fortgang der Angelegenheit sogleich abgeschnitten habe, indem ich Herrn Geheimrat Königsberger erklärt habe, dass ich einem Ruf nach Heidelberg unter keinen Umständen folgen würde. Ich habe dies gethan in dem Vertrauen, dass Euer Hochwohlgeboren mir das Wohlwollen, das Sie mir von dem ersten Augenblicke meiner Laufbahn an geschenkt haben, unvermindert bewahren werden, nicht minder auch in der festen Zuversicht, dass der Entschluss der Kgl. preussischen Regierung, Göttingen zu einer Centralstelle für die Pflege der mathematischen Wissenschaft nebst der Nachbarfächer zu machen, ein unerschütterlicher und für alle Zukunft dauernder bleiben wird.» Vgl. auch die Schreiben Althoffs an Hilbert vom 10. und 15. August 1904 in SUB Cod. Ms. Hilbert 4/10a resp. 4/13 und den Briefentwurf Hilberts an Althoff vom 22. August 1904 in SUB Cod. Ms. Hilbert 4/11, sowie Koenigsberger (1919) 206. Entsprechende Akten im Heidelberger Universitätsarchiv habe ich nicht auffinden können.

27 UAG PA Hilbert, Bl. 21-23; auch die Ernennung zum Geheimrat am 14. November 1904 (SUB Cod. Ms. Hilbert 743/7) steht in auffälliger zeitlicher Nähe zur Ablehnung des Heidelberger Rufes. 
Während des Ersten Weltkriegs ergab sich eine weitere Möglichkeit für Hilbert, durch Bleibeverhandlungen seine eigene Position und die der Göttinger Mathematik überhaupt zu stärken. Noch kurz vor Ausbruch des Krieges, am 22. Juni 1914, hatte die Philosophische Fakultät der Berliner Universität die Einrichtung eines vierten mathematischen Ordinariats beantragt ${ }^{28}$. Zur Begründung wurde in einem Kommissionsbericht die in den letzten zehn Jahren stetige Verkleinerung des mathematischen Lehrkörpers und das Alter der derzeitigen Ordinarien angeführt; man versäumte aber auch nicht, auf Göttingen hinzuweisen, das seit der Berufung Runges ja ebenfalls vier etatsmässige Ordinarien hätte. Den Auftrag des Ministers vorwegnehmend, schlug der Bericht für dieses Ordinariat an erster Stelle den damals 53jährigen Hilbert vor. Vermutlich wegen des Ausbruchs des Krieges blieb die Sache erst einmal liegen, bis der Minister die Fakultät zweieinhalb Jahre später, am 3. November 1916, unterrichtete, dass in Aussicht genommen sei, «in den Entwurf zum Staatshaushaltsetat für 1917 die Mittel zur Begründung eines Ordinariats als künftig wegfallend einzustellen», und mit Bezug auf den Bericht vom Juni 1914 um Personalvorschläge in der üblichen Dreizahl bat ${ }^{29}$. Die leicht erweiterte Kommission tagte daraufhin am 17. November 1916 und beschloss, an ihrem Vorschlag, Hilbert auf Platz 1 zu setzen, festzuhalten ${ }^{30}$. Die Fakultät stimmte zu, und der Bericht wurde am 19. Dezember 1916 an den Minister weitergeleitet ${ }^{31}$. Der Ruf wurde erteilt, und Anfang Januar kam Hilbert zu Verhandlungen nach Berlin. Abermals lehnte Hilbert ab, und am 15. Januar 1917 wurde eine mehrere Punkte umfassende Vereinbarung getroffen, welche die Bedingungen für sein Verbleiben in Göttingen festlegte ${ }^{32}$.

In dieser Vereinbarung wurde zunächst Hilberts Gehalt auf $8400 \mathrm{M}$ zuzüglich einer besonderen Besoldungszulage von $1100 \mathrm{M}$ festgesetzt, zusammen mit der Zusage, dass sich «nach Friedensschluß der Herr Kultusminister bemühen wird, bei dem Herrn Finanzminister eine Umwandlung dieser besonderen Besoldungszulage in Gehalt zu erreichen». Zusätzlich wurde Hilbert «vom ersten Jahre nach Friedensschluß ab» eine Kolleggeldgarantie von $4000 \mathrm{M}$ und für die Zeit vorher eine jährliche ausserordentliche Remuneration von $2000 \mathrm{M}$ bewilligt.

28 Biermann (1988) bes. 221-224, und Archiv der Humboldtuniversität Berlin, Phil. Fak. 1466, B1. 304-309.

29 Ebd., Phil. Fak. 1467, Bl. 124.

30 Ebd., Phil. Fak. 1467, Bl. 125.

31 Ebd., Phil. Fak. 1467, B1. 127-130.

32 GSPK Rep. 76 Va, Sekt. 6, Tit. IV, Nr. 1, Bd. XXV, Bl. 101. Eine Abschrift dieser Vereinbarung in Käthe Hilberts Hand (mit dem offensichtlich irrtümlichen Datum 15. Januar 1915) findet sich in SUB Cod. Ms. Hilbert 741/5. Vgl. auch UAG PA Hilbert, B1. 39. 
Interessant sind auch die weiteren Punkte dieser Vereinbarung. Hier ging es zunächst um Hilberts Assistenten. Hilbert war der erste Mathematiker, der bereits seit einigen Jahren zwei persönliche Assistenten beschäftigte. Hilbert, der in Forschung und Lehre in hohem Masse auf die Hilfe persönlicher Assistenten zurückgriff, hatte grosses Interesse daran, zwei Assistenten zu behalten. Nachdem er sich mehrere Jahre intensiv mit mathematischer Physik beschäftigt hatte, wollte er sich nun verstärkt der mathematischen Grundlagenforschung zuwenden und wünschte Unterstützung sowohl in der weiteren Bearbeitung der Fragen der theoretischen Physik als auch in der Beschäftigung mit der mathematischen Logik. Diesem Wunsch entsprechend wurden Hilbert weiterhin zwei Assistenten zugesichert, und es wurde darüber hinaus «in Aussicht genommen», dass auch der zweite Assistent eine Remuneration von 1200 M statt bisher nur 800 M bekommen solle. Aber nicht nur um diese persönlichen Assistenten ging es Hilbert. Offensichtlich hatte er in den Verhandlungen auch die Situation Emmy Noethers (18821935) angesprochen: Diese hatte sich mit Hilberts Unterstützung bereits zweimal - trotz einem 1908 ausdrücklich ergangenen Verbot der Habilitation von Frauen in Preussen - um die venia legendi in Göttingen beworben, war aber jedesmal an der konservativen Göttinger Fakultätsmehrheit gescheitert, so dass sie ihre Vorlesungen und Seminare nur unter Hilberts Namen ankündigen konnte ${ }^{33}$. Jedenfalls hält die Vereinbarung neben der Zusage der Assistenten fest: «Ferner wird in Aussicht genommen, nach dem Kriege Fräulein Nöther eine Remuneration zu gewähren, um sie dem Göttinger Seminar zu erhalten.»

Der folgende Punkt der Vereinbarung betrifft Hilberts Kollegen Debye (1884-1966), seit 1914 in Göttingen Ordinarius für Physik, mit dem Hilbert seit dem Wintersemester 1915/16 regelmässig gemeinsame Seminare über Struktur der Materie abhielt. Die Vereinbarung sah hier vor: «Die bisher von Professor Voigt gezahlte Summe von 2000 M. für einen erstklassigen Physiker in Göttingen wird, falls es Herr Voigt wünscht, jederzeit auf Zentralfonds übernommen werden.» Dahinter verbarg sich folgendes: Um Debye bei seiner Berufung nach Göttingen dessen Zürcher Gehalt bieten zu können, hatte Woldemar Voigt (1850-1919) sich bereit erklärt, das staatlich garantierte Maximaleinkommen aus seinem Privatvermögen noch zu erhöhen. Diese Regelung, die ohne Wissen Debyes erfolgt war, sollte im Sommer 1917 $\mathrm{zu}$ vorübergehenden Verstimmungen führen, als Voigt argwöhnte, dass Debyes zusätzliche Kolleggeldeinnahmen aus der Übernahme der «grossen»

33 Über Emmy Noethers Habilitation vgl. Tollmien (1991). 
Experimentalvorlesung sein Einkommen ohnehin in die von Voigt garantierte Höhe steigen lasse ${ }^{34}$.

Schliesslich enthält die Vereinbarung noch einen letzten Punkt, der den (Wieder-)Aufbau Göttingens zu einem internationalen Zentrum der mathematischen Physik nach Kriegsende betraf: «Herr Hilbert wünscht die Einrichtung von Gastprofessuren im Mathematisch-Physikalischen Seminar für die Sommer-Semester nach Friedensschluß.» Genauer gesagt, ging es hierbei um den Wunsch Hilberts, vor allem Niels Bohr (1885-1962) einmal für längere Zeit nach Göttingen einladen zu können. Ohne definitive Zusage wird auch dieser Wunsch Hilberts in der Vereinbarung aktenkundig: «Als Jahreskosten kämen dafür etwa $5000 \mathrm{M}$ in Frage. Ohne eine bindende Verpflichtung zu übernehmen, wird der Herr Minister sich bemühen, dahin zu wirken, daß sich diese Wünsche sei es im Rahmen der Gesellschaft der Wissenschaften, sei es im Geschäftskreis des Mathematisch-Physikalischen Seminars ermöglichen lassen.»

Mit dieser Vereinbarung vom 15. Januar 1917 hatte Hilbert sowohl seine persönliche Position als auch die Situation der mathematischen Forschung in Göttingen deutlich gefestigt. Er sass auf einem ausgezeichnet ausgestatteten Ordinariat an einer Universität, die seit zwei Jahrzehnten systematisch zu einem Zentrum der mathematischen Forschung in Preussen ausgebaut worden war. Sein oben erwähntes Gutachten vom 7. Dezember 1918 für den jungen Pólya, der vor dem Krieg drei Semester an dem mathematischen Leben Göttingens teilgenommen hatte, entsprach in seinem Ton und seinem Inhalt ganz und völlig der Position, die Hilbert in der mathematischen Welt erworben hatte.

\section{Der Ruf}

In seinem Brief an Regierungsrat Merz vom 28. Juni 1919 hatte Huber nicht nur von Schwierigkeiten bei der bisherigen Suche nach einem Nachfolger für Graf zu berichten ${ }^{35}$, sondern er konnte auch eine unerwartete Wendung in dieser Angelegenheit mitteilen. Es sei nämlich ein Brief des Göttinger Mathematikers Erich Hecke (1887-1947) eingegangen mit dem Hinweis,

dass wir bei einigem Zuwarten, infolge der traurigen politischen Verhältnisse in Deutschland, vielleicht die Möglichkeit hätten einen Mathematiker ersten Ranges nach Bern zu bekommen, der unter normalen Verhältnissen einen Ruf nicht annehmen würde.

34 Vgl. hierzu Voigt an den Minister, 18. Januar 1917, in GSPK Rep. 76 Va, Sekt. 6, Tit. IV, Nr. 1, Bd. XXV, Bl. 102-103; Bd. XXIV, Bl. 374f.; Voigt an Hilbert, 1. Juli 1917, in SUB Cod. Ms. Hilbert 415/5.

$35 \mathrm{Vgl}$. Fussnote 11. 
Tatsächlich, so führt Huber weiter aus, habe sich diese angedeutete Möglichkeit schneller als erwartet eingestellt:

Auf eine von Prof. Gruner an Herrn Debye, Prof. der math. Physik in Göttingen, gestellte Anfrage antwortete dieser, dass er als Nichtfachmann den ersten Vertreter der Math. in Göttingen, Herr Prof. Hilbert um Rat gefragt habe u. dass dieser, zu seiner Ueberraschung, sich geneigt gezeigt habe unter gewissen Bedingungen selbst einen Ruf nach Bern anzunehmen.

Interessanterweise berichtet Huber von einer ganz ähnlichen Reaktion des Mathematikers Constantin Carathéodory (1873-1950). Er fährt fort:

Ebenso wäre Karatheodory, Prof. der Math. in Berlin, der vor einem Jahr von Göttingen nach Berlin berufen worden war, nachdem Hilbert abgelehnt hatte, geneigt eine Berufung nach Bern anzunehmen.

Hubers Erklärung für diese unerwartete Wendung sind die allgemeinen Verhältnisse in Deutschland:

Berlin u. Göttingen sind die ersten Hochschulen in Deutschland für das Studium der Mathematik, letzteres seit dem grossen Mathematiker Gauss. An beiden Orten werden die math. Lehrstühle nur mit den hervorragendsten Mathematikern besetzt, u. wenn 2 derselben an unsere kleine Universität Bern übersiedeln möchten, so muss es schlimm aussehen in Deutschland.

Die Chance, das Problem der Nachfolge Graf nun doch auf so unerwartete Weise lösen zu können, ergriff Huber sofort beim Schopf. Nach Absprache mit den anderen Kommissionsmitgliedern sendete er umgehend, am 24. Juni, ein Telegramm nach Göttingen:

Würden Sie einen Ruf an die Universität Bern annehmen? Staatsbesoldung $10500 \mathrm{Fr} .{ }^{36}$.

Ob Hilbert dieses Telegramm erwartet hatte oder nicht, ist unklar. Aber bereits am 25. Juni reichte er es an den Göttinger Universitätskurator weiter und bat um eventuellen Urlaub für eine Reise nach Bern, falls der Ruf tatsächlich erfolgen sollte ${ }^{37}$. Der Urlaub wurde gewährt, und bereits am 26. Juni erhielt Huber telegraphisch die Antwort Hilberts ${ }^{38}$ :

Sobald Ruf erhalten, komme ich zur persönlichen Rücksprache.

Auf diese Antwort Hilberts hin beschloss die Kommission am 27. Juni, der Fakultät Hilbert als geeigneten Kandidaten vorzuschlagen. Am Tag darauf wandte sich Huber in dem bereits zitierten Brief direkt an Merz in dieser

36 Zitiert in Hubers Brief an Merz, vgl. Fussnoten 11 und 35. Huber offeriert hier das Maximalgehalt eines Berner Professors. Dieses war erst am 19. März 1919 in Reaktion auf die Geldentwertung neu festgesetzt worden und betrug jährlich $8500 \mathrm{Fr}$. plus Alterszulagen von jeweils 500 Fr., die bis zu dem angebotenen Maximalgehalt von 10500 Fr. alle drei Jahre gewährt wurden, vgl. «Dekret betreffend die Besoldungen der Professoren und Dozenten der Hochschule», Bern, 20. März 1919, und Feller (1935) 509-517.

37 UAG PA Hilbert, Bl. 50; GSPK Rep. 76Va, Sekt. 6, Tit. IV, Nr. 1, Bd. XXVI, Bl. 43.

38 Zitiert in Hubers Brief an Merz, vgl. Fussnote 11. 
Sache, und zwei Tage später beschloss die Fakultät, und zwar einstimmig, diesen Vorschlag der Kommission anzunehmen. Der Dekan benachrichtigte noch am gleichen Tag, am 30. Juni, die Unterrichtsdirektion über den Fakultätsbeschluss und bat darum, sich sofort mit Hilbert in Verbindung zu setzen und

nichts unversucht zu lassen, um für unsere Hochschule diesen Gelehrten von Weltruf, eine Kraft aller ersten Ranges, zu gewinnen ${ }^{39}$.

Falls gewünscht, würde man die Verdienste Hilberts in einem näheren Gutachten würdigen. Durch den Brief Hubers bereits über die Situation in Kenntnis gesetzt, entschied die Unterrichtsdirektion aber umgehend, vorerst ohne weiteres Gutachten den Ruf zu erteilen, und sandte Hilbert bereits am Vormittag des folgenden Tages ein Telegramm mit der offiziellen Mitteilung des einstimmigen Fakultätsbeschlusses und der Bitte, Hilbert möge telegrafieren, wann er «zu näherer Besprechung» nach Bern kommen könne. In seinem Antworttelegramm vom 4. Juli kündigte Hilbert einen Besuch noch «vor berner semesterschluss 24 . juli» an, da er «frueher amtlich nicht abkoemmlich» $\mathrm{Se}^{40}$.

Hilbert meldete die nun offizielle Erteilung des Rufes durch die Berner Erziehungsdirektion umgehend dem Kurator, und dieser wiederum verständigte sofort das Preussische Kultusministerium in Berlin. Noch am gleichen Tag, am 1. Juli, jedenfalls schrieb der Berliner Regierungsrat Wende an Hilbert und bat ihn, in jedem Falle eine Stellungnahme des Ministeriums abzuwarten, «da wir es natürlich sehr bedauern würden, Sie für Preussen zu verlieren $»^{4}$. Und am 3. Juli, also noch vor Hilberts Antwort nach Bern, erschien in der Göttinger Zeitung eine Notiz «Berufung Hilberts nach Bern», in der es heisst:

Wie uns kurz vor Redaktionsschluß aus Berlin gemeldet wird, hat Geheimrat Prof. Dr. Hilbert einen Ruf an die Universität Bern erhalten. Ueber die Stellungnahme des Gelehrten konnten wir bis zum Schluß des Blattes nichts mehr in Erfahrung bringen. Es ist dringend zu hoffen, dass es gelingt, Geheimrat Hilbert, der wohl als der hervorragendste Mathematiker der Jetztzeit gelten kann, unserer Universität zu erhalten ${ }^{42}$.

Über seine Motive mag sich Hilbert bedeckt gehalten haben, aber der nun erteilte Ruf war spätestens mit dieser Zeitungsnotiz öffentlich und lud

39 Otto Schulthess an Leo Merz, 30. Juni 1919, ebd.

40 Aus dem Telegrammstil geht nicht hervor, ob mit dem «24. juli» das Berner Semesterende gemeint sei, oder Hilberts Ankunft. In seinem Antwortkonzept an Wende (s. nächste Fussnote) nennt Hilbert jedoch den 24. Juli als Termin für seine Reise nach Bern. Das Göttinger Sommersemester ging laut Vorlesungsverzeichnis noch bis zum 15. August (Vorlesungen Göttingen 1919, p. 1).

41 Wende an Hilbert, 1. Juli 1919, SUB Cod. Ms. Hilbert 428a/1.

42 Göttinger Zeitung, Nr. 19161, vom 3. Juli 1919. 
zu Spekulationen ein. Wenige Tage später beispielsweise wandte sich der ursprünglich primo loco gesetzte, aber dann nicht berufene Karl Boehm brieflich an Hilbert, gratulierte ihm zur Berufung auf diese Stelle, auf die er sich ja auch Hoffnung gemacht habe, und äusserte den Wunsch, dass Hilbert ihn nun in irgendeiner Weise bei eventuellen bevorstehenden Stellenbesetzungen berücksichtigen möge, freilich nicht ohne hinzuzufügen, dass Hilbert den Inhalt dieses Briefes ja nicht unbedingt verbreiten müsse ${ }^{43}$. Auch in Zürich wusste man inzwischen von der Angelegenheit, und einen Tag später erhielt Hilbert einen Brief seines ehemaligen Studenten Hermann Weyl (1885-1955), seit 1913 Ordinarius für höhere Mathematik am Züricher Polytechnikum:

[...] so besteht wohl begründete Aussicht, dass Sie uns, vielleicht schon in der allernächsten Zeit, hier in Zürich die Freude Ihres Besuchs machen? Dass Sie ernsthaft mit Bern verhandeln, hat hier natürlich allgemeines Staunen hervorgerufen. Ich glaube ja schon Ihre Motive zu verstehen, und halte es auch für wahrscheinlich, dass die Dinge in Deutschland sich so entwickeln werden, dass die deutsche Schweiz im nächsten Jahrzehnt Zufluchtsort und Bewahrer der deutschen Kultur werden wird. Aber Sie sollten sich doch die Verhältnisse an der Berner Universität zunächst einmal genauer mit eigenen Augen anschauen; sehr verlockend, glaube ich, sind die Zustände da nicht ${ }^{44}$.

Die Berner Berufungsverhandlungen fanden dann vermutlich in der letzten Juliwoche statt ${ }^{45}$. Am 5. August unterrichtete Unterrichtsdirektor Merz Hilbert, der inzwischen nach Baden im Aargau gefahren war, in einem Brief über die zugesagten Bedingungen, die am gleichen Tag vom Regierungsrat genehmigt wurden ${ }^{46}$. Hilberts in Göttingen garantiertes Mindesteinkommen von $14200 \mathrm{M}$, wovon $10200 \mathrm{M}$ pensionsberechtigt waren ${ }^{47}$, wurde darin durch das Angebot eines Jahresgehalts von 15000 Fr. zuzüglich Kollegiengeldern und Examensgebühren sowie einem Ruhegehalt von 10000 Fr. überboten.

Mit diesem Angebot hatte die Erziehungsdirektion den ihr zustehenden Verhandlungsspielraum in der Tat vollständig ausgeschöpft. Denn wenn auch § 4 des im März 1919 erlassenen Besoldungsdekrets ${ }^{48}$ vorsah, dass das

43 Boehm an Hilbert, 11. Juli 1919, SUB Cod. Ms. Hilbert 32/3.

44 Weyl an Hilbert, 12. Juli 1919, SUB Cod. Ms. Hilbert 431/7; über Hermann Weyls diverse eigene Berufungsverhandlungen während seiner Zeit an der ETH Zürich vgl. Frei/Stammbach (1992).

45 Ein Treffen Hilberts mit Huber und Merz fand am 1. August statt, s. Huber an Merz, 24. August 1919, in SAB BBIIIb616, Nr. 16. Während seines Aufenthalts waren Hilbert und seine Frau, die ihn begleitete, zweimal bei Professor Moser und Gemahlin zu Gast, vgl. Moser an Hilbert, 17. September 1919, SUB Cod. Ms. Hilbert 265a/2.

46 Merz an Hilbert, 5. August 1919, ebd.

47 Hilberts Jahresgehalt von 14200 M setzte sich nach den Berliner Berufungsverhandlungen (s.o.) zusammen aus dem Gehalt von $8400 \mathrm{M}$, dem Wohnungszuschuss von $720 \mathrm{M}$, einer Besoldungszulage von $1100 \mathrm{M}$ «für die Mitbeteiligung an der Leitung des MathematischPhysikalischen Seminars» sowie einer Kolleggeldgarantie von $4000 \mathrm{M}$.

$48 \mathrm{Vgl}$. Fussnote 36. 
Maximalgehalt der Berner Ordinarien von 10500 Fr. in einzelnen Fällen erhöht werden könne, «um der Hochschule besonders hervorragende Lehrkräfte zu gewinnen oder zu erhalten», so waren lediglich vier Berner Professoren in den Genuss dieser Regelung gekommen, drei mit einem Gehalt von 11500 Fr., und einzig ein Ordinarius der juristischen Fakultät bezog ein gleichwertiges Spitzengehalt von 15000 Franken $^{49}$.

Über seine eigene Besoldung hinaus wurde Hilbert das Gehalt eines Assistenten in Höhe von 2500 Fr. bewilligt mit der Option, «den ausgesetzten Gehalt zu spalten, und damit 2 Assistenten zu dotieren», und es wurde die Absicht erklärt, Hilberts Wünschen in bezug auf die Bibliothek «nach Kräften entgegen zu kommen». In seinem Brief teilte Merz Hilbert schliesslich noch mit, bis wann er sich zu entscheiden habe:

Ihrer endgültigen Entschliessung sehen wir entsprechend unserer mündlichen Abmachung, bis zum 20. August nächsthin entgegen.

\section{Die Erwägung}

Nach Göttingen zurückgekehrt, hatte Hilbert nun zwei Wochen Zeit, sich zu entscheiden, ob er in die Schweiz gehen und Göttingen verlassen oder ob er den Ruf nach Bern ausschlagen solle. Welche Überlegungen mögen dabei eine Rolle gespielt haben ${ }^{50}$

Es ist natürlich möglich, dass Hilbert von vornherein nicht die Absicht hatte, nach Bern zu kommen, und seinen Namen nur ins Spiel gebracht hatte, um in etwaigen Bleibeverhandlungen seine Position in Göttingen weiter auszubauen. Andererseits spricht einiges dafür, dass Hilbert eine Annahme des Rufes ernsthaft in Erwägung zog ${ }^{51}$. Hilbert hatte seit vielen Jahren enge Verbindungen in die Schweiz. Schon seine besten Freunde aus Königsberger Zeit, Adolf Hurwitz und Hermann Minkowski, waren lange Jahre in Zürich

49 Vgl. hierzu SAB BBIIIb 521.

50 Hilberts Frau hatte ihren Mann auf seiner Reise nach Bern begleitet (vgl. Fussnote 45). Der Vollständigkeit halber sei bemerkt, dass mir keine Quellen bekannt sind, aus denen hervorginge, welche Einstellung Käthe Hilbert zu einem möglichen Wechsel nach Bern hatte.

51 Auch enge Freunde Hilberts vermuteten, dass Hilbert den Ruf ernsthaft erwog. So schreibt etwa der ehemalige Schüler und spätere Biograph Hilberts, Otto Blumenthal, nachdem er von Hilberts Ablehnung erfuhr, er habe während der schwebenden Berufung «ängstliche Stunden» verlebt: «Ich kann mir wohl vorstellen, dass Ihnen die Wahl schwer geworden ist, und dass Sie sich auch jetzt noch manchmal fragen werden, ob Sie das richtige getan haben.» Blumenthal an Hilbert, 30. August 1919, SUB Cod. Ms. Hilbert 30/43a. Tatsächlich äussert Hilbert in einem Brief an Einstein vom März 1920 sein Bedauern darüber, dass er den Ruf seinerzeit abgelehnt habe, Einstein Archiv Jerusalem, Nr. 13-067. 
gewesen $^{52}$, von dort kam auch sein jetziger Physikkollege Peter Debye. Kontakte nach Zürich bestanden auch über Richard Bär (1892-1940), der ab Ostern 1916 Hilberts physikalischer Assistent war und im Herbst 1917 zurück nach Zürich ging, aber weiterhin in engem freundschaftlichem Kontakt mit Hilbert blieb ${ }^{53}$. Ehemalige Schüler Hilberts, wie Hermann Weyl, Georg Pólya, Ernst Zermelo (1871-1953), um nur einige zu nennen, waren ebenfalls in die Schweiz gegangen. Seit vielen Jahren verbrachte Hilbert regelmässig die Semesterferien in der Schweiz, zumal seit Ausbruch des Ersten Weltkrieges. Diese Reisen waren für Hilbert Zeiten gesundheitlicher Erholung in besseren wirtschaftlichen Verhältnissen und besonders mit besserer Ernährung ${ }^{54}$. Auch das politisch liberale Klima der demokratischen, neutralen Schweiz sagte Hilbert im Gegensatz zum alldeutsch-monarchistisch geprägten Göttingen sehr zu, und er nutzte seine Aufenthalte in der Schweiz zu unzensierter Korrespondenz mit Kollegen aus Ländern des kriegsführenden Auslands ${ }^{55}$.

Anders als für viele seiner national-konservativen Kollegen war die politische Entwicklung Deutschlands nach dem Ende des Ersten Weltkrieges für Hilbert kein Grund zum Pessimismus. Er gehörte zu der Minderheit deutscher Akademiker, zumal in Göttingen, welche den Friedensschluss und die neue Demokratie begrüssten ${ }^{56}$. So hatte er bereits am 14. Oktober 1918, als das von vielen als Verrat und Niederlage abgelehnte, deutsche Waffenstillstandsangebot erklärt worden war, sein Kolleg über partielle Differentialgleichungen mit folgender Erklärung eröffnet:

Wenn also irgendwo Bedrücktheit oder Niedergeschlagenheit aufkommt, wir wollen den Frieden freudig hinnehmen, weil er uns unsere geliebte math. Arbeit wieder ermöglicht, deren Betätigung für uns die Befriedigung unseres Ideals ist und zugleich für unser Land und Volk von höchstem Nutzen u. zur Wi[e]dererlangung seiner geistigen und materiellen Stellung nötig ist ${ }^{57}$.

52 Minkowski war vor seiner Berufung nach Göttingen von 1896-1902 Professor für Höhere Mathematik an der ETH Zürich; Adolf Hurwitz (1859 - 18. November 1919) war seit 1892 Ordinarius an der ETH, über Hilberts Verhältnis zu diesen Mathematikern vgl. seine Nachrufe auf Hurwitz und Minkowski in Hilbert (1932-1935) Bd. 3, einen Überblick über die Mathematiker an den Zürcher Hochschulen gibt Frei/Stammbach (1994).

53 Vgl. die Korrespondenz Bärs mit Hilbert in SUB Cod. Ms. Hilbert 11.

54 Noch im September 1918 hatte sich Hilbert in Baden (Aargau) einer «vierwöchentlichen Kur gegen Rheumatismus (chronischer Hexenschuss)» unterzogen und hatte beim Kurator der Göttinger Universität vom Park-Hotel Vitznau aus um Verlängerung seines Urlaubs bis 10. Oktober gebeten, Hilbert an Osterrath, 22. September 1918, UAG PA Hilbert, Bl. 46-47.

55 Vgl. etwa die Korrespondenz Hilberts mit Bertrand Russell in SUB Cod. Ms. Hilbert 339.

56 Über das intellektuelle Klima in Göttingen zur Zeit des Ersten Weltkrieges und der Novemberrevolution vgl. Tollmien (1993) und Dahms (1988).

57 SUB Cod. Ms. philos. 182: D. Hilbert; eine Kopie befindet sich in der Ausarbeitung «Partielle Differentialgleichungen, Sommer 1916» im Mathematischen Institut der Universität Göttingen. 
In den neuen politischen Verhältnissen sah Hilbert durchaus neue Chancen, etwa für die nun endlich mögliche Habilitation Emmy Noethers ${ }^{58}$. Freilich mögen der Ausgang der Wahlen zur Nationalversammlung vom März 1919 und die Bedingungen des Versailler Friedensvertrages Hilberts ursprünglichen Optimismus zu dieser Zeit sehr gedämpft haben. Allgemein wurden die Möglichkeiten der ökonomischen Entwicklung in Deutschland sehr pessimistisch eingeschätzt. Selbst sein konservativer Kollege Voigt äusserte Verständnis für die Verlockung des Rufes nach Bern und gab in einem Brief an Hilbert sogar zu, dass bei ihm selbst manchmal der Wunsch aufkäme, nach seiner bevorstehenden Emeritierung «ins Ausland zu gehen». Aber er sehe doch für sich die Nachteile einer Verpflanzung in «ganz fremden Boden» und dagegen die Vorteile Göttingens und wolle Hilbert «recht herzlich bitten, die Übelstände, die Sie hier störend empfinden nicht allzu hoch einzuschätzen». In diesem Zusammenhang sah sich Voigt auch veranlasst zu versichern, dass «Widersprüche [...] im Kreise der Fachkollegen [...] immer sachliche Motive haben und unsere Wertschätzung für Sie nicht trüben» ${ }^{59}$.

Die Verbundenheit Hilberts mit der Göttinger Universität und eine Verpflichtung dem grossen, gemeinsamen Ziel gegenüber, Göttingen zu einer «Centralstelle für die Pflege der mathematischen Wissenschaft ${ }^{60}$ zu machen, worauf sich implizit auch Voigt bezieht, mögen für Hilbert ein ideeller Grund gewesen sein, den Berner Ruf abzulehnen ${ }^{61}$. Auch bot ihm das in Bern erwartete Lehrangebot weniger Gelegenheit für eigene Forschung, als dies in Göttingen möglich war ${ }^{62}$. Wenn auch Grafs Pensum von 22 Wochenstunden Unterricht ein freiwilliges Engagement für die Ausbildung gewesen sein mag, so wäre Hilbert in Bern mit dem Abhalten elementarer Anfängervorlesungen in viel stärkerem Masse belastet gewesen. Selbst während des redu-

58 Emmy Noethers Habilitationsverfahren wurde - im dritten Anlauf - am 4. Juni 1919 abgeschlossen, nachdem Felix Klein auf Anregung von Einstein (und sicherlich in Absprache mit Hilbert) am 5. Januar 1919 beim Ministerium in Berlin angefragt hatte, wie man sich unter den veränderten politischen Bedingungen zu einer Habilitation Noethers stellen würde (Tollmien [1991] 24-26).

59 Voigt an Hilbert, 9. August 1919, SUB Cod. Ms. Hilbert 415/6.

$60 \mathrm{Vgl}$. Fussnote 26.

61 Über Hilberts Verhältnis zur Göttinger Universität vgl. auch Hilbert (1971).

62 Auf diesen Punkt hebt Huber in seiner (nachträglichen) Interpretation der Verhandlungen mit Hilbert ab: «[...] dass er nur einige wenige Vorlesungen über ganz spezielle Kapitel der Math. - seine Lieblingsgebiete - zu halten geneigt war [...]. Dies ist allerdings an grossen Universitäten, wie Göttingen u. Berlin, der Brauch, aber bei uns in Bern, wo in der höhern Math. nur 2 Hauptvertreter, ein Geometer u. ein Analytiker vorhanden sind, sollte eben der letztere doch alle in sein Gebiet einschlagenden Hauptvorlesungen im Laufe von 4-6 Semestern abhalten, sonst bekommen unsere Studierenden keine vollständige Uebersicht über das ganze Gebiet der Math., u. kommen zu keinem richtigen Studienabschluss; Assistenten können die Lücke nicht ausfüllen. Auch Doktoranden hätte Herr Hilbert wahrscheinlich nicht gerne übernommen.» Huber an Merz, 24. August 1919, SAB BBIIIb 616, Nr. 16. 
zierten Lehrbetriebs während des Krieges hatte Hilbert in Göttingen fast ausschliesslich Vorlesungen über spezielle Themen der mathematischen Physik anbieten können. Und in seinen Bleibeverhandlungen nach dem Berliner Ruf hatte er für die Zeit nach Friedensschluss eine stattliche Kolleggeldgarantie aushandeln können, so dass er bei der Wahl seiner Vorlesungen auch keine Rücksicht auf materielle Überlegungen nehmen musste ${ }^{63}$.

Hinzu kam die Tatsache, dass ein Umzug nach Bern für Hilbert mit erheblichen Vermögensverlusten verbunden gewesen wäre. Hilbert hatte sich mit den Jahren nicht nur den Bau seines Göttinger Wohnhauses, sondern auch ein beachtliches Vermögen in Form von diversen Wertpapieren erspart ${ }^{64}$. Hinzu war 1907 eine Erbschaft beim Tode seines Vaters gekommen, und auch seine Frau hatte aus ihrer Familie Aktienkapital ererbt ${ }^{65}$. Tatsächlich machten die Zinserträge des Hilbertschen Kapitalvermögens im Jahre 1918 rund die Hälfte seines versteuerten Gesamteinkommens von etwas mehr als $26000 \mathrm{M}$ aus. Ein im Juli 1918 erlassenes «Gesetz gegen die Steuerflucht», das durch weitere Verordnungen gegen die «Kapitalabwanderung in das Ausland» auch noch nach der Annahme des Waffenstillstands ergänzt worden war ${ }^{66}$, bestimmte nun aber, dass Angehörige des Deutschen Reiches, die ihren Wohnsitz im Deutschland aufgeben, im vollen Umfang ihrer bis dahin geleisteten Steuern in Deutschland weiterhin steuerpflichtig blieben. Den Steuerbehörden mussten dafür vor dem Verlassen des Landes Sicherheiten in Höhe von $20 \%$ des gesamten Vermögens geleistet werden.

Vor dem Hintergrund dieser möglichen Erwägungen mag daher die Entscheidung über die Annahme oder Ablehnung des Rufes von möglichen Zugeständnissen des Berliner Ministeriums in Bleibeverhandlungen abgehangen haben. Mit dem Berner Berufungsangebot in der Tasche reiste Hilbert jedenfalls Mitte August nach Berlin zu Verhandlungen mit dem Berliner Kultusministerium. Dort war inzwischen ein Telegramm des Göttinger Universitätsrektors Reichenbach eingegangen:

63 Im Wintersemester 1918/19 las er lediglich zweistündig über Mengenlehre sowie einstündig und allgemeinverständlich über «Raum und Zeit». Seine vierstündige Vorlesung über «Partielle Integral- und Differentialgleichungen» sowie die zugehörigen zweistündigen Übungen hatte er dagegen zusammen mit Emmy Noether angekündigt, und das Seminar über Struktur der Materie hielt er gemeinsam mit Debye ab (Vorlesungen Goettingen [1918] 14-16).

64 Eine Übersicht des Hilbertschen Vermögens an Wertpapieren findet sich in SUB Cod. Ms. Hilbert 748, «Vermögen und Gehalt», B1. 26-33.

65 Für die Steuererklärung 1917 etwa hatte Hilbert sich die Höhe seines Vermögens zu 249245 M notiert. Dieses Vermögen setzte sich aus $161945 \mathrm{M}$ an Hypotheken, Anleihen und Obligationen, $42300 \mathrm{M}$ an Aktien sowie einem Schätzwert seines Hauses in Höhe von 45000 M zusammen, ebd. Bl. 10, 33.

66 «Gesetz gegen die Steuerflucht», Reichsgesetzblatt 1918, Nr. 100, S. 951ff. Einen zeitgenössischen Überblick über die Gesetzeslage bis 1920 gibt Gierke (1921). 
universitaet goettingen legt allergroeszten wert auf verbleiben von profeszor hilbert. bittet, wenn irgend moeglich, verhandlungen in diesem sinne zu entschejden ${ }^{67}$.

Am 13. August trafen Minister Naumann und Hilbert schliesslich eine Vereinbarung ${ }^{68}$ über die Bedingungen einer Ablehnung des Rufes. Darin wird zunächst einmal Hilberts Gehalt fast verdoppelt auf jährlich $25000 \mathrm{M}$, ohne Anrechnung von Fakultäts- und Prüfungsgebühren und mit einem Pensionsanspruch von $15000 \mathrm{M}^{69}$. Ausdrücklich festgelegt wird eine Klausel über Teuerungszulagen und eine Zusage, dass das Gehalt bei eventuellen Steuererhöhungen entsprechend angehoben wird. Die bereits in den letzten Bleibeverhandlungen zugesagten $5000 \mathrm{M}$ für eine Gastprofessur sollen nun dem Mathematisch-Physikalischen Seminar direkt zur Verfügung gestellt werden. Im letzten Punkt der Vereinbarung wird Hilbert zugesichert, dass bezüglich seiner Assistenten der Versuch unternommen werden soll, im Staatshaushaltsplan von 1920 eine etatsmässige Stelle zu begründen, wobei einem der Assistenten eine jährliche Vergütung von $1500 \mathrm{M}$ gewährt werden soll.

Die Vereinbarung hatte allerdings einen Haken. Die zugesagte Erhöhung des Grundgehalts ging über das normale Höchstgehalt der ordentlichen Professoren in Göttingen deutlich hinaus und konnte daher nicht vom Unterrichtsminister allein ausgehandelt werden, sondern bedurfte der ausdrücklichen Genehmigung des Finanzministers.

\section{Die Entscheidung}

Da Hilbert sich bis zum 20. August zu entscheiden hatte, telegrafierte er am 18. August nach Berlin:

erbitte hoeflichst mittejlung ob der herr minister die verabredungen vom letzten Mittwoch angenommen hat ${ }^{70}$.

Zwei Tage später entwarf der Kultusminister einen persönlichen Brief an seinen Kollegen vom Finanzministerium ${ }^{71}$. Es drohe dem Lehrkörper der

67 Reichenbach an das Ministerium für Wissenschaft, Kunst und Volksbildung, 2. Aug. 1919, GSPK Rep. 76Va, Sekt. 6, Tit. IV, Nr. 1, Bd. XXVI, Bl. 44.

68 GSPK Rep.76Va, Sekt. 6, Tit. IV,Nr.1, Bd. XXVI, Bl. 75; eine Kopie findet sich auch in UAG Pa Hilbert, Bl. 53.

69 Dieses Gehalt setzt sich zusammen aus dem Grundgehalt von $12000 \mathrm{M}$, dem Wohnungszuschuss von $720 \mathrm{M}$, einer Entschädigung für die Leitung des Math.-phys. Seminars von $2500 \mathrm{M}$, sowie «gewährleisteten Einnahmen an Vorlesungshonoraren» von $7780 \mathrm{M}$ plus 2000 M für die «Mitwirkung bei Ausfüllung von Lücken im Lehrkörper».

70 GSPK Rep. 76Va, Sekt. 6, Tit. IV, Nr. 1, Bd. XXVI, B1. 72.

71 Ebd., Bl. 45. 
Göttinger Universität ein schwerer Verlust, denn der Göttinger Professor Hilbert habe einen Ruf an die Universität Bern erhalten, der mit erheblichen finanziellen und sonstigen Vorteilen verbunden sei. Es wird das angebotene Jahresgehalt von 15000 Fr. genannt und noch erläutert, «dass gegenüber dem Stande unserer Valuta dieser Betrag um mehr als das Doppelte zu bewerten ist». Er verwies auf das unterstützende Telegramm des Göttinger Rektors und schloss sich den Wünschen der Universität «auf das wärmste» an; denn Hilbert sei «der erste Mathematiker Deutschlands, wenn nicht der Welt», wie der Minister handschriftlich noch hinzufügt. Hilbert wirke seit 1895 ununterbrochen an der Göttinger Universität und habe mit seiner Wirksamkeit in Lehre und Forschung wesentlich zum Aufschwung der Mathematik und Physik beigetragen. Er ersuche daher

ergebenst, sich hiernach gefälligst damit einverstanden zu erklären, dass dem Prof. Hilbert vom 1. Oktober d. Js. ab ein das normale Höchstgehalt der ordentlichen Professoren an der Univ. Göttingen um 3600 M übersteigendes Gehalt von jährlich 12000 M gewährt werde.

Bitten dieser Art betrafen den Stellenwert der universitären Wissenschaft im Gesamtbudget. Eine schriftliche Antwort auf dieses Gesuch erfolgte zehn Tage später ${ }^{72}$, in dem der Finanzminister seinen Kollegen daran erinnerte, dass er darauf hingewiesen habe

welch enge Schranken der Betätigung des Staates in finanzieller Hinsicht gesteckt sind. Nur dann kann der völlige Zusammenbruch der Staatsfinanzen vermieden werden, wenn auf allen Gebieten ohne Ausnahme äusserste Sparsamkeit und Zurückhaltung geübt wird. Die Wissenschaft und die Universitäten würden sich deshalb einer Verkennung des hohen Ernstes der Lage hingeben, wenn sie in finanzieller Richtung etwa glauben, dem allgemeinen Schicksal der Nation entzogen zu sein und nicht auch ihre Ansprüche auf ein sehr bescheidenes Mass zurückführen zu müssen. Im übrigen vermag ich, da zu dem normalen Höchstgehalt der ordentlichen Universitätsprofessoren z.Zt. noch die Teuerungszulagen und die wohl noch für einige Zeit sehr erheblich gesteigerten Kollegiengelder hinzukommen, nicht anzuerkennen, dass dieses Einkommen nicht genügen sollte, tüchtige Lehrkräfte den Preussischen Universitäten zu erhalten.

Ungeachtet dieser allgemeinen Überlegungen wolle er gleichwohl Hilberts Gehaltserhöhung auf $12000 \mathrm{M}$ bewilligen, bitte aber «auf weitere Zugeständnisse in dieser Richtung nicht mehr zu rechnen ${ }^{73}$.

Vor dieser schriftlichen Bestätigung hatten sich die Kollegen Minister bereits mündlich geeinigt, denn Hilberts Telegramm vom 18. August trägt den handschriftlichen Vermerk: «Die Verabredungen vom letzten Mittwoch sind vom Herrn Minister genehmigt worden. 20. 8. 19.»74 Einen Tag später, am 21. August, telegraphierte Hilbert nach Bern:

72 Ebd., Bl. 73.

73 Ebd. Im gleichen Brief wurde eine weitere Ausnahme für den Bonner Professor Spiethoff bewilligt, dessen Gehalt auf $11000 \mathrm{M}$ erhöht wurde.

74 Siehe Fussnote 70. 
Noch am gleichen Tage unterrichtete der Unterrichtsdirektor Merz den Dekan Schulthess über die Absage und bemerkte:

er war mit allem einverstanden, hätte aber gern mit Rücksicht auf die Vermögensverluste, die der Umzug nach der Schweiz mit sich bringen könnte, eine Erhöhung des festen Gehaltes gewünscht. Ich erklärte ihm weshalb ich nicht weiter gehen könne, und er anerkannte, dass der Betrag auch nach deutschen Verhältnissen gemessen, reichlich sei. Er erklärte mir, dass er, bevor er sich definitiv über die Berufung äussern könne, noch mit dem Ministerium in Berlin Rücksprache nehmen müsse und ich vermute, dass seine Absage darauf zurückzuführen ist $^{76}$.

In dem offiziellen Ablehnungsschreiben Hilberts an Merz vom 22. August nannte Hilbert nur die finanziellen Überlegungen als den ausschlaggebenden Grund für die Ablehnung:

Trotz des weitgehenden Entgegenkommens Ihrerseits bin ich bei reiflicher Ueberlegung zu der Ueberzeugung gekommen, dass die pekuniären Verhältnisse sich für mich in Bern zu ungünstig gestalten würden, da ich wesentlich nur auf das Gehalt angewiesen wäre ${ }^{77}$.

\section{Hilbert fügte hinzu:}

So nehme ich schweren Herzens von dem Wunsche Abschied, während meines ferneren Lebens in der herrlichen Schweiz zu wirken, wo ich so Viele meiner jungen Freunde und Schüler ihr Glück finden sah.

Nach Berlin schrieb Hilbert eine Woche später:

Euer Exzellenz sende ich beiliegend das eine Exemplar der Vereinbarung vom 13. d. M. unterschrieben zurück. Für den weggehenden Kollegen Hecke hoffen wir vollgültigen Ersatz zu erhalten und auch in jeder anderen Hinsicht zu erreichen, dass Göttingen auf der alten Höhe bleibt.

Mit wiederholtem Dank und den ergebensten Grüssen

Hochachtungsvoll

Hilbert ${ }^{78}$.

\section{Das Ergebnis}

Sobald Huber von der Ablehnung erfuhr, schrieb er umgehend an Merz, bedauerte diesen Entschluss, bemerkte aber zugleich, dass ihn das Treffen mit Hilbert in Bern «einigermassen enttäuscht» habe, denn dieser sei ihm doch für sein Alter «ziemlich gebrechlich» vorgekommen. Auch dass Hilbert nur über seine Spezialgebiete habe Vorlesungen halten wollen, habe ihn schon bei den Verhandlungen mit Sorge erfüllt.

75 Hilbert an Merz, 21. August 1919, SAB BBIIIb 616, Nr. 16.

76 Merz an Schulthess, 21. August 1919, ebd.

77 Hilbert an Merz, 22. August 1919, ebd.

78 GSPK Rep. 76Va, Sekt. 6, Tit. IV, Nr. 1, Bd. XXVI, Bl. 74. 
Hubers Sorge wies auf das nun wieder offene, drängende Problem der mathematischen Ausbildung an der Berner Hochschule hin. Sein Brief an Merz endet mit der klagenden Feststellung:

Ich hätte nicht geglaubt, dass die Neubesetzung dieser Mathematikprofessur so viele Schwierigkeiten machen würde ${ }^{79}$.

Nach weiterer Kandidatensuche ${ }^{80}$ und nach nochmals erfolglosen Verhandlungen mit Rudolf Fueter aus Zürich entschied man sich schliesslich, auf eine bereits im ersten Kommissionsbericht vom Februar 1919 erwogene Alternative zurückzugreifen, und schlug im Februar 1920, ein Jahr später, der Unterrichtsdirektion vor, Jacques Louis Crelier den Lehrauftrag Grafs zu übergeben, der damit dessen Nachfolge antreten solle, und den frei werdenden Lehrauftrag dem Zürcher Extraordinarius Ferdinand Gonseth durch Berufung zum Ordinarius für Geometrie zu übertragen.

Mit der Annahme dieses Vorschlags durch Beschluss des Regierungsrats vom 4. März 1920 war die Nachfolge Graf, knapp zwei Jahre nach dessen Tod, entschieden ${ }^{81}$.

\author{
Abkürzungen \\ PFB Universität Bern, Philosophisch-historische Fakultät \\ SAB Staatsarchiv des Kantons Bern \\ GSPK Geheimes Staatsarchiv Preussischer Kulturbesitz Berlin \\ SUB Niedersächsische Staats- und Universitätsbibliothek Göttingen, Handschriften- \\ abteilung \\ UAG Universitätsarchiv Göttingen
}

\title{
Literatur
}

Bieberbach, Ludwig (1930). - «Über den Einfluss von Hilberts Pariser Vortrag über «Mathematische Probleme> auf die Entwicklung der Mathematik in den letzten dreissig Jahren», Die Naturwissenschaften 18(51), 1101-1111.

79 Huber an Merz, 24. August 1919, SAB BBIIIb 616, Nr. 16.

80 Hilbert selbst hatte in seinem Absagebrief (s. Fussnote 77) als Ersatz für seine eigene Person erstens Felix Hausdorff, zweitens Richard Courant und drittens Karl Boehm vorgeschlagen. Wie sich die Kommission zu diesen Vorschlägen gestellt hat, ist allerdings nicht dokumentiert.

81 Nach Abschluss der Korrektur an diesem Aufsatz hat der Hilbert-Nachlass in der Staats- und Universitätsbibliothek Göttingen einen bedeutsamen Zuwachs erfahren. Glücklichen Bemühungen Klaus Sommers ist es gelungen, einige verschollen geglaubte und grösstenteils unbekannte Korrespondenzen Hilberts aufzufinden und dem Göttinger Nachlass zuzufügen. Für das Thema dieses Aufsatzes ist aus diesem Fund ein Brief Max Borns vom 27. Jan. 1919 von Interesse, in dem die Motive Hilberts aus Borns Sicht kommentiert werden und hier insbesondere die «Stellung zu den Göttinger Kollegen, die grösstenteils dem Alldeutschtum anhängen» angeführt wird. Ich danke Klaus Sommer für diesen Hinweis. 
Biermann, Kurt-R. (1988). - Die Mathematik und ihre Dozenten an der Berliner Universität 1810-1933: Stationen auf dem Wege eines mathematischen Zentrums von Weltgeltung. Berlin: Akademie-Verlag.

Blumenthal, Otto (1935). - «Lebensgeschichte», in: David Hilbert, Gesammelte Abhandlungen 3, Berlin/Heidelberg/New York: Springer, 388-429.

Brocke, Bernhard vom (1980). - «Hochschul- und Wissenschaftspolitik in Preussen und im Deutschen Kaiserreich 1882-1907: das «System Althoff»», in: Peter Baumgart (Hrsg.), Bildungspolitik in Preussen zur Zeit des Kaiserreichs, Stuttgart: Klett-Cotta, 9-118.

Corry, Leo (1999). - «From Mie's Electromagnetic Theory of Matter to Hilbert's Unified Foundations of Physics», Stud. Hist. Phil. Mod. Phys. 30 (2), 159-183.

Dahms, Hans-Joachim/Frank Halfmann (1988). - «Die Universität Göttingen in der Revolution von 1918/19», in: Hans-Georg Schmeling (Hrsg.), 1918. Die Revolution in Südhannover, Göttingen: Städtisches Museum Göttingen, 59-82.

Feller, Richard (1935). - Die Universität Bern 1834-1934, Bern: Paul Haupt.

Frei, Günther/U. Stammbach (1992). - Hermann Weyl und die Mathematik an der ETH Zürich, 1913-1930, Basel/Boston/Berlin: Birkhäuser.

Frei, Günther/U. Stammbach (1994). - Die Mathematiker an den Zürcher Hochschulen, Basel/ Boston/Berlin: Birkhäuser.

Gierke, Julius von (1921). - «Berichte über die deutschen Reichsgesetze der Jahre 1919 und 1920. I. Handelsrecht», Zeitschrift für das Gesamte Handelsrecht und Konkursrecht 85, 434-455.

Gorgé, Viktor (1984). - «Die Entwicklung der exakten Wissenschaften an der Berner Hochschule. Ein Beispiel des Professionalisierungsprozesses», in: Kommission für bernische Hochschulgeschichte (1984b) 319-351.

Hilbert, David (1912). - Grundzüge einer allgemeinen Theorie der linearen Integralgleichungen, Leipzig/Berlin: Teubner.

Hilbert, David (1915). - «Die Grundlagen der Physik. (Erste Mitteilung.)», Nachrichten der Königlichen Gesellschaft zu Göttingen. Math.-phys. Klasse, 395-407.

Hilbert, David (1932-1935). - Gesammelte Abhandlungen 1-3, Berlin: Springer.

Hilbert, David (1971). - «Über meine Tätigkeit in Göttingen», in: Kurt Reidemeister (Hrsg.), Hilbert Gedenkband, Berlin/Heidelberg/New York: Springer, 78-82.

Hilbert, David (1998). - The Theory of Algebraic Number Fields. Translated from the German by Iain T. Adamson. With an Introduction by Franz Lemmermeyer and Norbert Schappacher, Berlin/Heidelberg/New York: Springer.

Hilbert, David (1999). - Grundlagen der Geometrie, 14. Auflage, hrsg. u. mit Anhängen versehen von M. Toepell, Leipzig: Teubner.

Koenigsberger, Leo (1919). - Mein Leben, Heidelberg: Winters Universitätsbuchhandlung.

Kommission für bernische Hochschulgeschichte (Hrsg.) (1984a). - Die Dozenten der bernischen Hochschule. Ergänzungsband zu: Hochschulgeschichte Berns 1528-1984, Bern: Universität Bern.

Kommission für bernische Hochschulgeschichte (Hrsg.) (1984b). - Hochschulgeschichte Berns 1528-1984, Bern: Universität Bern.

Reid, Constance (1970). - Hilbert, Berlin/Heidelberg/New York: Springer.

Renn, Jürgen/J. Stachel (1999). - Hilbert's Foundation of Physics: From a Theory of Everything to a Constituent of General Relativity, Max Planck Institute for the History of Science Berlin, Preprint 118.

Rowe, David (1989). - «Klein, Hilbert, and the Göttingen Mathematical Tradition», Osiris 5, 186-213.

Sauer, Tilman (1999). - «The Relativity of Discovery: Hilbert's First Note on the Foundations of Physics», Arch. Hist. Exact. Sci. 53, 529-575.

Scharlau, Winfried (1989). - Mathematische Institute in Deutschland 1800-1945, Braunschweig: Vieweg.

Sommerfeld, Arnold/C. Carathéodory (1943). - «Zum Andenken an David Hilbert. Gestorben am 14. Februar 1943. Ansprachen im Trauerhause am Morgen des Begräbnistages vor dem Sarge», Die Naturwissenschaften 31(19/20): 212-213.

Tobies, Renate (1991). - «Wissenschaftliche Schwerpunktbildung: der Ausbau Göttingens zum Zentrum der Mathematik und Naturwissenschaften», in: Bernhard vom Brocke (Hrsg.), Wissenschaftsgeschichte und Wissenschaftspolitik im Industriezeitalter, Hildesheim: Lax, 87-108. 
Tollmien, Cordula (1991). - «Die Habilitation von Emmy Noether an der Universität Göttingen», NTM-Schriftenr. Gesch. Naturw., Techn., Med. Leipzig 28(1),13-32.

Tollmien, Cordula (1993). - «Der «Krieg der Geister in der Provinz - das Beispiel der Universität Göttingen 1914-1919», in: Göttinger Jahrbuch 1993, 137-210.

Verzeichnis Bern (1918). - Verzeichnis der Behörden, Lehrer, Anstalten und Studierenden der Universität Bern im Sommersemester 1918, Bern: Buchdrucker Emil Horat.

Vorlesungen Bern (1918). - Universität Bern. Vorlesungen im Sommersemester 1918, Bern: Buchdruckerei Dr. Gustav Grunau.

Vorlesungen Goettingen (1918). - Verzeichnis der Vorlesungen auf der Georg-August-Universität zu Göttingen während des Winterhalbjahres 1918/19, Göttingen: Dieterich'sche Universitätsdruckerei.

Vorlesungen Göttingen (1919). - Verzeichnis der Vorlesungen auf der Georg-August-Universität zu Göttingen während des Sommerhalbjahres 1919, Göttingen: Dieterich'sche Universitätsdruckerei.

Weyl, Hermann (1944). - «David Hilbert and his Mathematical Work», Bulletin of the American Mathematical Society 50, 612-654.

Wussing, Hans (Hrsg.) (1976). - Die Hilbertschen Probleme, Leipzig: Akademische Verlagsgesellschaft Geest \& Portig. 